\title{
HARMONIE-AROME, MODELO OPERATIVO DE ESCALA CONVECTIVA DE AEMET. PARTE II: ASIMILACIÓN DE DATOS
}

\author{
Maria Diez, Jana Sanchez, Joan Campins \\ AEMET, c/ Leonardo Prieto Castro s/n, 28040 Madrid \\ mdiezm@aemet.es
}

\section{Resumen}

Desde junio de 2017, HARMONIE-AROME es el nuevo modelo operativo de alta resolución de AEMET. Es un modelo no-hidrostático que resuelve la convección profunda explícitamente y que se integra con una resolución horizontal de $2,5 \mathrm{~km}$. Los modelos de predicción como éste, constan de un sistema de asimilación de datos que mejora sustancialmente la calidad de los pronósticos, ya que permite evaluar el estado futuro de la atmósfera partiendo del mejor estado inicial posible.

\section{INTRODUCCIÓN}

Es de sobra conocida la importancia de las condiciones iniciales en la calidad de las predicciones obtenidas por los modelos de predicción numérica del tiempo. Tal es así que se debe empezar a ejecutar el modelo a partir del estado atmosférico más cercano a la realidad. Para conseguir dicho objetivo se han desarrollado técnicas de asimilación de datos cada vez más complejas, donde existe un equilibrio entre su complejidad y el tiempo necesario para ejecutarlas, de manera que las salidas del modelo numérico se generen a tiempo para que puedan usarse por los predictores en los centros de predicción y vigilancia. También es necesario tener un sistema de telecomunicaciones robusto para que las observaciones puedan recibirse a tiempo y que puedan ser asimiladas en el modelo numérico.

En AEMET se ejecuta 8 veces al día el modelo HARMONIE-AROME, para los dos dominios operativos, uno que cubre la península ibérica y las islas Baleares y el otro que cubre las islas Canarias (en adelante AIB y AIC). En todas las pasadas se genera un análisis con las observaciones disponibles, sobre el cual se inicializa el modelo.

\section{ALGORITMOS DE ASIMILACIÓN}

Existen múltiples algoritmos de asimilación de datos, como se puede comprobar en Gustafsson et. al (2018) pero para el modelo HARMONIE-AROME se ha demostrado que el uso de la Asimilación Variacional Tridimensional (3D-VAR) es uno de los más eficientes. Los algoritmos variacionales se basan en la teoría de Bayes de probabilidad condicionada. Dado unas observaciones $\mathrm{y}_{0}$, y un campo previo (llamado background) la probabilidad a posteriori de obtener la distribución del valor "real" de una variable atmosférica es:

$$
p\left(x \mathrm{I} y_{0}\right)=\frac{p\left(y_{0} \mid x\right) p_{B}(x)}{p\left(y_{0}\right)}
$$


Donde:

$$
\begin{array}{r}
p_{B}(x)=\frac{1}{(2 \pi)^{n / 2}|B|^{1 / 2}} e^{-1 / 2\left[\left(x_{B}-x\right)^{T} B^{-1}\left(x_{B}-x\right)\right]} \\
p\left(y_{0} \mid x\right)=\frac{1}{(2 \pi)^{n / 2}|R|^{1 / 2}} e^{-1 / 2}\left[\left(y_{0}-H(x)\right)^{T} R^{-1}\left(y_{0}-H(x)\right)\right]
\end{array}
$$

Siendo R la matriz de covarianza de los errores de la observación, B la matriz de covarianza de los errores del Background (o campo previo) y $\mathrm{H}(\mathrm{x})$ el operador de observación.

Por lo tanto la probabilidad de obtener el valor de una variable analizada es:

$$
p\left(x_{A}\right)=C * e^{-1 / 2}\left[\left(y_{0}-H(x)\right)^{T} R^{-1}\left(y_{0}-H(x)\right)+\left(x_{B}-x\right)^{T} B^{-1}\left(x_{B}-x\right)\right]
$$

Según el principio de máxima verosimilitud, el valor del análisis es el que haga máximo $p\left(x_{A}\right)$. Máximizar $p\left(x_{A}\right)$ es equivalente a minimizar $-\ln \left(p\left(x_{A}\right)\right)$, siendo esta expresión igual a la función coste $J$. Como observamos en la fig. 1 publicada por Bouttier (1999), la función coste se forma con un término relativo al campo previo $J b_{(x)}$ y otro término debido a las observaciones $J o_{(x)}$ y el mínimo de la función $J$ es posible que sea mayor que el de sus términos por separado.

$$
J=\left(y_{0}-H(x)\right)^{T} R^{-1}\left(y_{0}-H(x)\right)+\left(x_{B}-x\right)^{T} B^{-1}\left(x_{B}-x\right)
$$

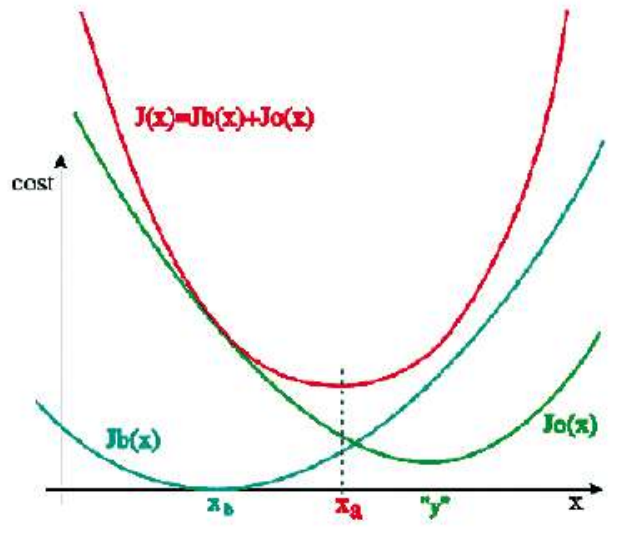

Figura 1: Representación del mínimo de la función de coste $\mathrm{J}(\mathrm{x})$ para el punto $\mathrm{x}_{\mathrm{a}}$ que constituye el valor del análisis.

El valor mínimo de J se obtiene al igualar el gradiente de $\mathrm{J}$ a 0 . La manera más eficiente de calcular dicho valor mínimo es mediante un proceso iterativo, tal y como muestra Holm (2008) en la fig. 2. 


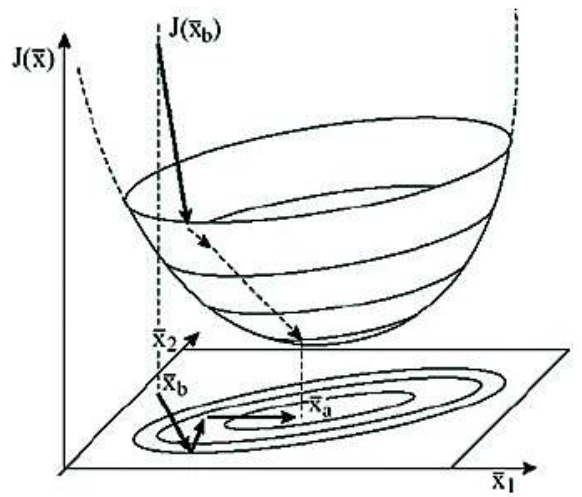

Figura 2: Representación del proceso iterativo para alcanzar la mejor solución de la función de coste $\mathrm{J}_{(\mathrm{x})}$.

\section{USO DE OBSERVACIONES}

Los tipos de observaciones que se usan en la asimilación operativa de HARMONIE-AROME en AEMET son: las estaciones convencionales en superficie, (synop, ship, buoy) las observaciones de radiosondeos (temp), observaciones tomadas en sensores integrados en el fuselaje de los aviones comerciales (airep), y observaciones provenientes de satélites polares.

Figura 3: Tipos de observaciones usadas en HARMONIE-AROME.

\section{Recepción de observaciones.}

Los diferentes boletines se reciben en la Sede Central de AEMET por medio del Sistema Mundial de Telecomunicaciones (GTS), y de EuMetCast, que es un sistema de diseminación de observaciones de satélite basado en los satélites geoestacionarios. El tiempo de espera para que se reciban todas las observaciones es de una hora.

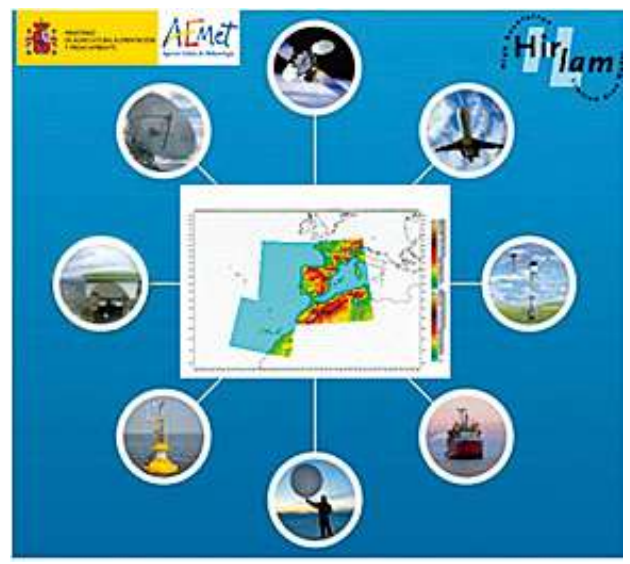

Observaciones de satélite.

Sabemos que las observaciones de satélite tanto los ATOVS como los GNSS presentan cierto sesgo, que depende en el caso de los ATOVS de los diferentes canales y de los satélites y en el caso de los GNSS de las diferentes estaciones. De acuerdo con el método de Dee (2005) y de Auligné (2007) se realiza una reducción de bias "dinámico" (Var-BC).

Var-BC introduce una selección de predictores previamente seleccionados, de manera que dichos predictores contribuyen en mayor o menor medida a explicar el bias asociado a una observación (Lindskog, 2012). Por lo que el operador de observación H, pasaría a tener una contribución del bias:

$$
H \sim\left(x_{i} \beta\right)=H(x)+\sum_{i=0}^{N} \beta_{i} p_{i}(x)
$$

Donde $\boldsymbol{p}_{i}(\boldsymbol{x})$ son los diferentes predictores y $\boldsymbol{\beta}_{i}$ los coeficientes que se deben ir adaptando en cada ciclo. 


\section{GNSS. ZTD}

Las observaciones ZTD (Zenith Total Delay) se obtienen midiendo el retraso en la señal recibida por el sistema satelital Global Navigation Satellite System (GNSS). Dicho retraso está relacionado con el contenido de humedad específica en la columna atmosférica. En la fig. 4 vemos los receptores GNSS distribuidos en uno de los dominios operativos. Como se puede observar es una red demasiado densa y es necesario reducir el número de estaciones usadas.

Las variables atmosféricas que muestran un mayor impacto al asimilar dichas observaciones son la precipitación y la humedad relativa en altura.

Figura 4: Representación típica de la distribución de las estaciones GNSS dentro del dominio de integración de la península ibérica

\section{ATOVS: AMSU-A \& AMSU- B MHS.}

Los datos ATOVS (Advanced TIROS Operational Vertical Sounder), se recogen de dos instrumentos diferentes AMSU-A (Advanced Microwave Sounder Unit - A) y AMSU - B (Advanced Microwave Sounder Unit - B) aunque este último instru-

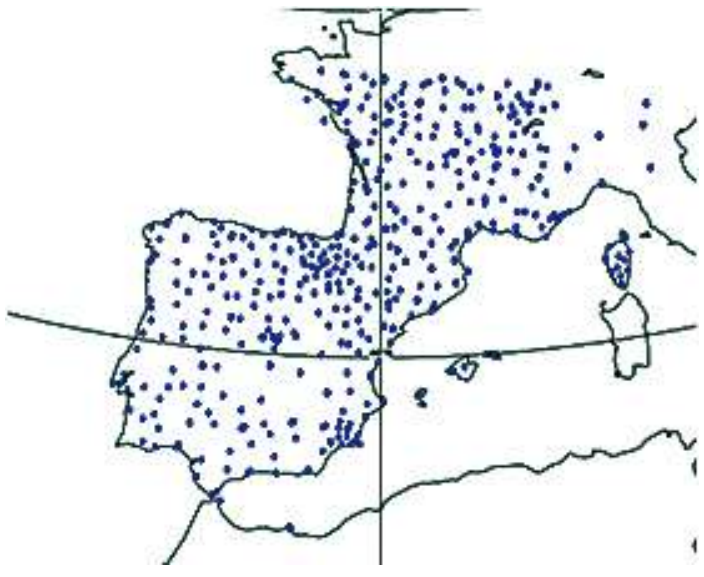
mento se está sustituyendo por el MHS (Microwave Humidity Sounder).

AMSU-A está equipado con 15 canales, los cuales miden la radiancia desde la superficie hasta el tope de la atmósfera, dichos canales se corresponden con diferentes longitudes de ondas. Once de los quince canales son sensibles a la temperatura en diferentes capas de la atmósfera. Mientras que el resto son sensibles a la temperatura de la superficie. Los canales útiles en la asimilación del modelo HARMONIE-AROME son 6, 7, 8 y 9. Los canales del 10 al 15, nos dan información de una zona de la atmósfera demasiado alta como para poder ser asimiladadas en nuestro modelo, con un tope de la atmosfera relativamente bajo, así mismo los canales superficiales tampoco son útiles para la asimilación operativa.

Los instrumentos AMSU-B y MHS, al igual que el AMSU-A miden la radiancia desde la superficie hasta el tope de la atmósfera en 5 canales. En este caso, las radiancias son sensibles al perfil de la humedad. Dos de los cinco canales son sensibles a la humedad en superficie, mientras que los tres más altos son sensibles únicamente al perfil atmosférico, son estos últimos los que asimilan.

Dichos instrumentos se encuentran a bordo de los satélites NOAA-18, NOAA-19, METOP-A y METOP-B. Gracias a dicha constelación es posible que se asimilen datos de satélite en prácticamente todos los ciclos de asimilación. 
Al igual que los datos GNSS, el impacto positivo de asimilar dichas observaciones se aprecia en el perfil de las variables de humedad y en la precipitación, también a veces en temperatura y presión. Estas conclusiones están más detalladas en Campins, et al (2017).

\section{CONCLUSIONES}

Partir del mejor estado inicial posible es una de las claves de la predicción numérica del tiempo, en los modelos que se ejecutan en los centros operativos de predicción se debe tener un balance entre el tiempo que debe tardar en generarse los ficheros de predicción y las diferentes técnicas, a su vez debe garantizarse la recepción de las observaciones a un tiempo adecuado.

Actualmente las observaciones de satélite son una fuente de información muy valiosa y con un gran potencial, a pesar de que son observaciones que presentan sesgo. Un procedimiento que ha resultado muy adecuado es el Var-BC, ya que permite disminuir el sesgo dinámicamente al incluir un término relativo al mismo en la función de coste de la asimilación variacional.

Otras fuentes de observación como la reflectividad de la red de radares, los datos de los satélites geostacionarios y más observaciones de otros instrumentos a bordo de los satélites polares anteriormente citados, se están intentando introducir en la pasada operativa del modelo HARMONIE-AROME de AEMET, donde se espera que se obtengan tan buenos resultados como los dos anteriormente descritos.

\section{BIBLIOGRAFÍA}

- Auligné, T., McNally A. P., Dee D. P. (2007) Adaptative bias correction for satellite data in a numerical weather prediction system. Q. J. R. Meteorol. Soc., 133, 631-642.

- Bouttier, F., Courtier, P., (1999) Data assimilation concepts and methods. ECMWF Data Assimilation Lecture Notes.

- Campins, J., Sanchez, J., Diez, M., Calvo, J., Navascués, B., Assimilation of ATOVS and GNSS ZTD data in the HARMONIE-AROME model configuration run at AEMET. (2017) ALADIN-HIRLAM Newsletter, 8, 40-51.

- Dee, D. P. (2005) Bias and Data Assimilation. Q. J. R. Meteorol. Soc., 131, 3323-3343.

- Gustafsson, N. , Janjić, T. , Schraff, C. , Leuenberger, D. , Weissmann, M. , Reich, H. , Brousseau, P. , Montmerle, T. , Wattrelot, E. , Bučánek, A. , Mile, M. , Hamdi, R. , Lindskog, M. , Barkmeijer, J. , Dahlbom, M. , Macpherson, B. , Ballard, S. , Inverarity, G. , Carley, J. , Alexander, C. , Dowell, D. , Liu, S. , Ikuta, Y. and Fujita, T. (2018) Survey of data assimilation methods for convective-scale numerical weather prediction at operational centres. Q.J.R. Meteorol. Soc.. . doi:10.1002/qj.3179

- Hólm, F. V., (2008) Lecture notes on assimilation algorithms. ECMWF Data Assimilation Lecture Notes.

- Lindskog, M., Dahlbom, M., Thorsteinsson, S., Dahlgren, P., Randriamampianina, R., Bojarova, J. (2012), ATOVS processing and usage in the HARMONIE reference system. Hirlam Newsletter, 59. 